\title{
SEARCH FOR BASIC PROPERTIES OF THE NUCLEON-NUCLEON INTERACTION
}

\author{
A. Gersten \\ TRIUMF and Department of Physics, University of British Columbia \\ Vancouver, B.C., Canada V6T 2A6 \\ and Department of Physics, Ben-Gurion University, Beer-Sheva, Israel
}

I. Introduction

Most commonly the $\mathrm{N}-\mathrm{N}$ interaction is analysed via potential or pseudopotential models at low and intermediate energies and through Regge trajectory exchanges and various diffraction models at high energies. In this work the $\mathrm{N}-\mathrm{N}$ amplitudes are obtained directly from phase-shift analysis.

From one point of view our aim is to check whether some features, evident in the direct amplitude analysis, are not omitted in the commonly used models. We also wish to check if some new relations exist for the $N-N$ amplitudes which can lead to a simplified analysis of the $N-N$ interaction. For example, it is possible that new relations or symmetries exist which will limit the number of independent amplitudes. In recent years new $\mathrm{N}-\mathrm{N}$ experiments were performed and phase-shift analyses up to $800 \mathrm{MeV}$ lab energy are available. ${ }^{l}$ It seems that by going to higher energies the real part of the amplitudes becomes better approximated by the first Born term. In this way one can get an insight into the meson exchange structure of the $\mathrm{N}-\mathrm{N}$ amplitudes. We would like to emphasize a significant difference in the present and the potential model approach. The potential models fit the experimental data up to about $400 \mathrm{MeV}$ lab energy, while the information we extract from our analysis comes mainly from the 400-800 MeV experiments.

In Sections II and III we give some new formulations of facts which are most probably known but not directly exposed in the literature.

\section{The number of independent amplitudes}

Let us consider first the $N-N$ helicity amplitudes of isospin $I=0$ and $I=1$ : $\left.<\lambda_{3} \lambda_{4}\left|T^{I}(E)\right| \lambda_{1} \lambda_{2}\right\rangle$, where $E$ is the total c.m. energy of one nucleon, $\lambda$ is the helicity equal to $\pm \frac{1}{2}$. Parity conservation, time reversal invariance, and charge independence reduce the 16 possible helicity amplitudes (for each value of the isospin) to 5 independent ones. ${ }^{2}$ This way one can construct 10 linearly independent amplitudes

$$
\begin{aligned}
& \phi_{1}^{I}=\left\langle\frac{1}{2} \frac{1}{2}\left|T^{I}(E)\right| \frac{1}{2} \frac{1}{2}\right\rangle, \phi_{2}^{I}=\left\langle\frac{1}{2} \frac{1}{2}\left|T^{I}(E)\right|-\frac{1}{2}-\frac{1}{2}\right\rangle, \phi_{3}^{I}=\left\langle\frac{1}{2}-\frac{1}{2}\left|T^{I}(E)\right| \frac{1}{2}-\frac{1}{2}\right\rangle, \\
& \phi_{4}^{I}=\left\langle\frac{1}{2}-\frac{1}{2}\left|T^{I}(E)\right|-\frac{1}{2} \frac{1}{2}\right\rangle, \phi_{5}^{I}=\left\langle\frac{1}{2} \frac{1}{2}\left|T^{I}(E)\right| \frac{1}{2}-\frac{1}{2}\right\rangle .
\end{aligned}
$$

From the Pauli principle the following non-Zinear dependence results ( $\theta$ is the c.m. scattering angle):

$$
\begin{aligned}
& \phi_{i}^{I}(\pi-\theta)=(-1)^{I+1} \phi_{i}^{I}(\theta), \quad i=1,2 ; \\
& \phi_{3}^{I}(\pi-\theta)=(-1)^{I} \phi_{4}^{I}(\theta) ; \quad \phi_{b}^{I}(\pi-\theta)=(-1)^{I} \phi_{5}^{I}(\theta) .
\end{aligned}
$$


Let us construct the following amplitudes:

$$
\phi_{i}^{n p}(\theta)=\frac{1}{2}\left[\phi_{i}^{O}(\theta)+\phi_{i}^{l}(\theta)\right], \quad i=1, \ldots, 5,
$$

which are the neutron-proton scattering amplitudes. From Eqs. (2.2) and (2.3) we obtain the following relations

$$
\begin{aligned}
& \phi_{i}^{I}(\theta)=\phi_{i}^{n p}(\theta)+(-1)^{I+1} \phi_{i}^{n p}(\pi-\theta), i=1,2, \\
& \phi_{i}^{I}(\theta)=\phi_{i}^{n p}(\theta)+(-1)^{I} \phi_{i}^{n p}(\pi-\theta), i=3,4,5 .
\end{aligned}
$$

From Eq. (2.4) we deduce that all the information about the $\mathrm{N}-\mathrm{N}$ amplitudes is contained in the five amplitudes $\phi_{i}^{n p}(\theta)$ from which the ten linearly independent amplitudes $\phi_{i}^{0}(\theta)$ and $\phi_{i}^{l}(\theta)$ can be derived. Another relation of 1 imited consequence is

$$
\phi_{i}^{n p}(-\theta)=\phi_{i}^{n p}(\theta), i=1,2,3,4 ; \quad \phi_{5}^{n p}(-\theta)=-\phi_{5}^{n p}(\theta),
$$

which may reduce the number of independent amplitudes to 4 . For example, one can construct

from which we obtain

$$
\phi_{15}^{n p}(\theta)=\frac{3}{2}\left[\phi_{1}^{n p}(\theta)+\phi_{5}^{n p}(\theta)\right] \text {, }
$$

$$
\phi_{1}^{n p}(\theta)=\phi_{15}^{n p}(\theta)+\phi_{15}(-\theta) ; \quad \phi_{5}^{n p}(\theta)=\phi_{15}^{n p}(\theta)-\phi_{15}(-\theta) .
$$

Equation (2.5) may have an application in the study of ambiguities of phase-shift analysis. ${ }^{3}$ Using Eq. (2.4) for $\theta=0$ and replacing isospin $I=1$ by $p p$ or $n n$ scattering we have

$$
\phi_{i}^{n p}(\pi)=\phi_{i}^{p p}(0)-\phi_{i}^{n p}(0), i=1,2 ;-\phi_{4}^{n p}(\pi)=\phi_{3}^{p p}(0)-\phi_{3}^{n p}(0) .
$$

Taking the imaginary parts of Eq. (2.7) we obtain an extension of the optical theorem to backward angles

$$
\begin{aligned}
& \frac{2 \pi}{p} \operatorname{Im}\left[\phi_{1}^{n p}(\pi)-\phi_{4}^{n p}(\pi)\right]=\sigma_{\text {tot }}^{p p}-\sigma_{\text {tot }}^{n p} \\
& -\frac{4 \pi}{p} \operatorname{Im} \phi_{2}^{n p}(\pi)=\Delta \sigma p p-\Delta \sigma \sigma_{T}^{n p} \\
& \frac{4 \pi}{p} \operatorname{Im}\left[\phi_{1}^{n p}(\pi)+\phi_{4}^{n p}(\pi)\right]=\Delta \sigma_{L}^{p p}-\Delta \sigma_{L}^{n p} .
\end{aligned}
$$

\section{The Fermi amplitudes}

In order to investigate the meson exchange content of the scattering amplitude it will be advantageous to use the decomposition of the scattering matrix in terms of the Fermi invariants ${ }^{2} S, V, T, A, P$. At a given energy

$$
T=a_{S}(t) S+a_{V}(t) V+a_{T}(t) T+a_{A}(t) A+a_{P}(t) P,
$$

where $t$ is the Mandelstam variable. We shall relate the Fermi amplitudes $a_{S}, a_{V}, a_{T}$, $a_{A}$, ap to the helicity amplitudes. As the direct relations are well known we shall concentrate on inverting them. We shall write the direct relations in the following new way

$$
\begin{aligned}
& \alpha \phi_{1}=A_{1}+4 B A_{2}+S_{1} Z ; \alpha \phi_{2}=-A_{1}+B A_{3}+\left(S_{1}+B S_{2}\right) Z ; \\
& \alpha\left(\phi_{3}-\phi_{4}\right)=B A_{4}+\left(2 S_{1}+B S_{3}\right) Z ; \\
& \alpha\left(\phi_{3}+\phi_{4}\right)=2 S_{1}+B S_{3}+B A_{4} Z ; \alpha \phi_{5}=-E \sin \theta S_{1} / m,
\end{aligned}
$$

where $\beta=p^{2} / m^{2}, Z=\cos \theta, p$ is the $c . m$. momentum, $m$ is the nucleon mass, $\alpha=2$ if the $\phi_{i}$ are normalized according to Eq. (2.8), and 


$$
\begin{aligned}
& A_{1}=a_{S}+a_{V}-3 a_{T}-3 a_{A} ; \quad A_{2}=a_{V}-a_{A} ; \quad A_{3}=-a_{S}+6 a_{T}-a_{P} \\
& S_{1}=a_{S}+a_{V}+a_{T}+a_{A} ; \quad S_{2}=a_{S}+2 a_{T}+a_{P} \\
& A_{4}=-a_{S}+2 a_{V}+2 a_{A}+a_{P}=4 A_{2}-2 A_{1}-A_{3} \\
& S_{3}=a_{S}+2 a_{V}+2 a_{A}-a_{P}=2 S_{1}-S_{2} .
\end{aligned}
$$

Comparing Eqs. (3.2) and (2.2) we see that the intermediate amplitudes $A_{i}$ and $S_{i}$ have the following symmetry properties resulting from the Pauli principle

$$
A_{i}^{I}(\pi-\theta)=(-1)^{I+1} A_{i}^{I}(\theta) ; \quad S_{i}^{I}(\pi-\theta)=(-1)^{I} S_{i}^{I}(\theta),
$$

which are equivalent to Eq. (2.2). We shall find the inverse relations in two stages

$$
\begin{aligned}
& S_{1}=-\alpha m \phi_{5} /(E \sin \theta) ; \quad A_{1}=S_{1} Z+\alpha m^{2}\left(\phi_{1}-\phi_{2}-\phi_{3}+\phi_{4}\right) /\left(2 E^{2}\right) \\
& A_{2}=m^{2}\left(\alpha \phi_{1}-A_{1}-S_{1} Z\right) /\left(4 p^{2}\right) ; \quad A_{4}=\alpha m^{2}\left[\phi_{3} /(1+Z)-\phi_{4} /(1-Z)\right] / p^{2} \\
& S_{3}=m^{2}\left[\alpha \phi_{3} /(1+Z)+\alpha \phi_{4} /(1-Z)-2 S_{1}\right] / p^{2}
\end{aligned}
$$

and

$$
\left(\begin{array}{l}
a_{S} \\
a_{V} \\
a_{T} \\
a_{A} \\
a_{p}
\end{array}\right)=\frac{1}{8}\left(\begin{array}{rrrrr}
2 & -4 & -1 & 6 & -1 \\
0 & 4 & 1 & 0 & 1 \\
-2 & 4 & -1 & 2 & -1 \\
0 & -4 & 1 & 0 & 1 \\
2 & -4 & 3 & 6 & -5
\end{array}\right)\left(\begin{array}{l}
A_{1} \\
A_{2} \\
A_{4} \\
S_{1} \\
S_{3}
\end{array}\right)
$$

\section{The meson exchange content of the Fermi amplitudes}

The meson exchange content of the Fermi amplitudes is complicated due to the presence of exchange diagrams. Let us denote by $D$ a Feynman diagram of one-meson exchange (direct diagram) and by $\mathcal{E}$ the exchange diagram. The one-meson exchange contribution to $p-p$ scattering is given by $\mathcal{D}+\mathcal{E}$, while in the case of $n-p$ scattering we have $-D+2 \mathcal{E}$ for isovector meson exchange and $D$ for isoscalar meson exchange. The one-meson exchange contribution to the lowest order direct Feynman diagram acquires the following form

$$
b_{i}(t)= \pm m^{2} g_{i}^{2} /\left[4 \pi E\left(t-\mu_{i}^{2}\right)\right],
$$

where the sign depends on the particular type of the interaction, $g$ is the coupling constant and $\mu$ the meson mass. The exchange diagrams can be related to the direct ones by the matrix of the fierz transformation. For instance, if we assume onemeson exchanges for $p-p$ scattering, the Fermi amplitudes will have the following form:

$$
\left(\begin{array}{l}
a_{S}(t) \\
a_{V}(t) \\
a_{T}(t) \\
a_{A}(t) \\
a_{P}(t)
\end{array}\right)=\left(\begin{array}{l}
b_{S}(t) \\
b_{V}(t) \\
b_{T}(t) \\
b_{A}(t) \\
b_{P}(t)
\end{array}\right)-\frac{1}{4}\left(\begin{array}{rrrrr}
-1 & -4 & -6 & -4 & -1 \\
-1 & 2 & 0 & -2 & 1 \\
-1 & 0 & 2 & 0 & -1 \\
-1 & -2 & 0 & 2 & 1 \\
-1 & 4 & -6 & 4 & -1
\end{array}\right)\left(\begin{array}{l}
b_{S}(u) \\
b_{V}(u) \\
b_{T}(u) \\
b_{A}(u) \\
b_{P}(u)
\end{array}\right),
$$

where $t=-2 p^{2}(1-\cos \theta), u=-2 p^{2}(1+\cos \theta)$.

Let us write Eq. (4.2) in the matrix form

$$
\vec{a}(t)=\vec{b}(t)-F \vec{b}(u) \text {, }
$$

where $F$ is the matrix of the Fierz transformation. Let us assume for the moment that 
the Fermi amplitudes can also be represented in the form of Eq. (4.3). In this way we can try to resolve the meson exchange content of the Fermi amplitudes. From Eq. (4.3) we have

$$
\vec{a}(u)=\vec{b}(u)-F \vec{b}(t) \text {. }
$$

The amplitudes $\vec{a}(t)$ and $\vec{a}(u)$ can be obtained from the phase-shift analysis. Thus Eqs. (4.3) and (4.4) together consist of 10 equations with 10 unknowns, $\vec{b}(t)$ and $\vec{b}(u)$. Unfortunately 5 of the 10 equations are dependent because of Eq. (3.4). Thus it is impossible to resolve the meson exchange content of the Fermi amplitudes in a simple way. Instead, we shall get information on the meson exchange content by generating particular combinations of the Fermi amplitudes.

We shall concentrate on two combinations of the isospin amplitudes:

(i) $T P P+T^{n P}=1 / 2 T^{0}+3 / 2 T^{1}$ - for this choice the isovector meson exchange is cancelled in the forward direction. We shall call this combination the isoscalar amplitude.

(ii) $T^{P p}-T^{n P}=-1 / 2 T^{\circ}+1 / 2 T^{1}-$ here the isoscalar meson exchange is cancelled in the forward direction. We shall call this combination the isovector amplitude. This is also the so-called charge exchange amplitude.

Instead of analysing the isovector amplitude in the forward direction one can analyse the np amplitude in the backward direction. Notice that the np amplitudes have exchange diagrams only for isovector exchanges.

\section{Energy-independent results}

Below we display two figures in which the results become stable with the increase of energy. Let us introduce the notation

$$
\gamma=\frac{1}{2} E \cdot \operatorname{Re}\left[a_{j}(0)\right], j=S, V, T, A, P,
$$

with $i=0$ for isoscalar amplitudes and $i=1$ for isovector amplitudes. These quantities are simply related to the one-meson-exchange contributions at zero momentum transfer

$$
\gamma \simeq \pm m_{N}^{2} g_{j}^{2} F_{j}^{2}(0) /\left(4 \pi \mu_{j}^{2}\right),
$$

where $\mu_{j}$ is the meson mass, $g_{j}$ the coupling constant and $F_{j}(0)$ the value of the form factor at zero momentum transfer. Our results are based on a recent phase-shift analysis of Arndt and VerWest ${ }^{1}$ in which the higher partial waves $(J>6)$ are represented by the one-pion-exchange (OPE) phase shifts with the pseudoscalar coupling constant $g^{2} / 4 \pi=14.5$. In Fig. I the energy dependence of the $\gamma_{j}^{i}$ is given. As one can see, except for $\gamma_{p}^{\circ}$, all $\gamma_{j}^{i}$ become more or less stable above $500 \mathrm{MeV}$ lab energy. There are four large quantities: $\gamma_{p}^{1}$ related to the pion exchange, $\gamma_{V}^{0}$ related to $\omega$ and $\phi$ exchange, $\gamma_{S}^{0}$ related to the $\varepsilon$ exchange, and finally the energy-unstable $\gamma_{p}^{0}$. There is one surprise in the above results: there is no large contribution which can be associated with the $\rho$ exchange.

Figure 2 is related to the $\pi N N$ form factor. For this purpose we consider the backward np scattering for which all the Fermi amplitudes are dominated by the pion pole. 


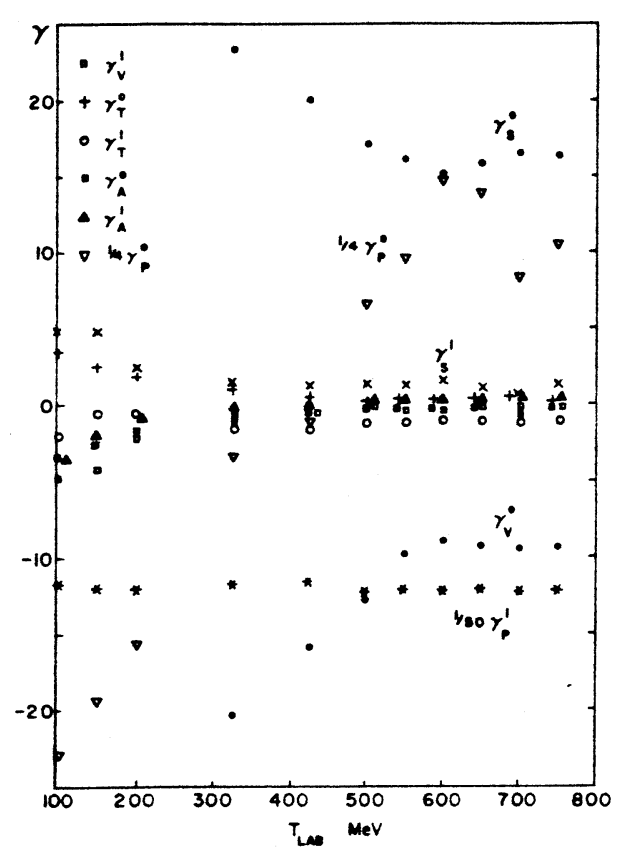

Fig. 1

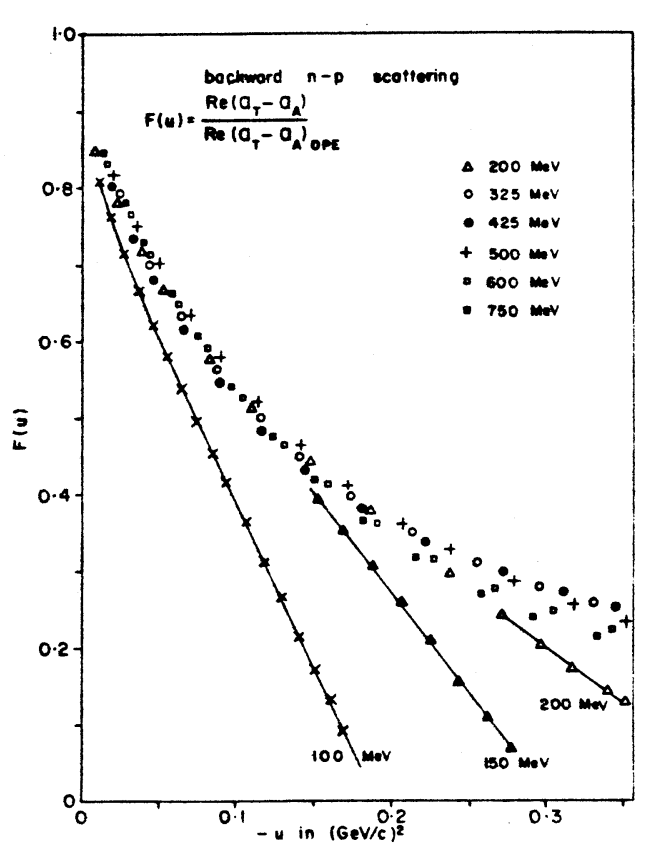

Fig. 2

We should avoid taking amplitudes which are large in the forward direction as such amplitudes may have still a residual large contribution in the backward direction. One test of an absence of this influence will be a consistent $u$ dependence and energy independence of our result. As shown in Fig. 2 it seems that the combination $a_{T}^{n p}(u)-a_{A}^{n p}(u)$ satisfies this criterion. Above $200 \mathrm{MeV}$ lab energy this curve is found to be independent of energy. One cannot interpret the result obtained in terms of the $\pi N N$ form factor only, because of the contribution of the two-pion-exchange cut. However, this cut can be approximated by the conventional $\rho$-meson contribution.

Let $\beta$ be the slope of the curve formed in Fig. 2 near the pion pole $\left[\beta \simeq 5(\mathrm{GeV} / \mathrm{c})^{-2}\right]$. We can compare this value with that of some models of the $\pi N N$ form factor if we include an approximation for the $\rho$ exchange. Let us take, for example, the form factor derived on the basis of the cloudy bag model. ${ }^{4}$ Comparing slopes near the pion pole we obtain in the non-relativistic approximation:

$$
B \simeq R^{2} /\left(5+m_{\pi}^{2} R^{2} / 7\right)+\left(g_{\rho}+f_{\rho}\right)^{2} /\left[g_{\pi}^{2}\left(m_{\rho}^{2}-m_{\pi}^{2}\right)\right] \simeq R^{2} / 5+\left(g_{\rho}+f_{\rho}\right)^{2} /\left(g_{\pi}^{2} m_{\rho}^{2}\right),
$$

where $R$ is the radius of the bag, $g_{\rho}$ and $f_{\rho}$ are the electric and magnetic $\rho$ coupling constants, respectively. If we substitute $g_{\pi}^{2} / 4 \pi=14.5$, vector meson dominance model values $g_{\rho}^{2} / 4 \pi=0.6, f_{\rho} / g_{\rho}=3.7$ and $m_{\rho}=765 \mathrm{MeV}$, we obtain $R \simeq 0.8 \mathrm{fm}$. 

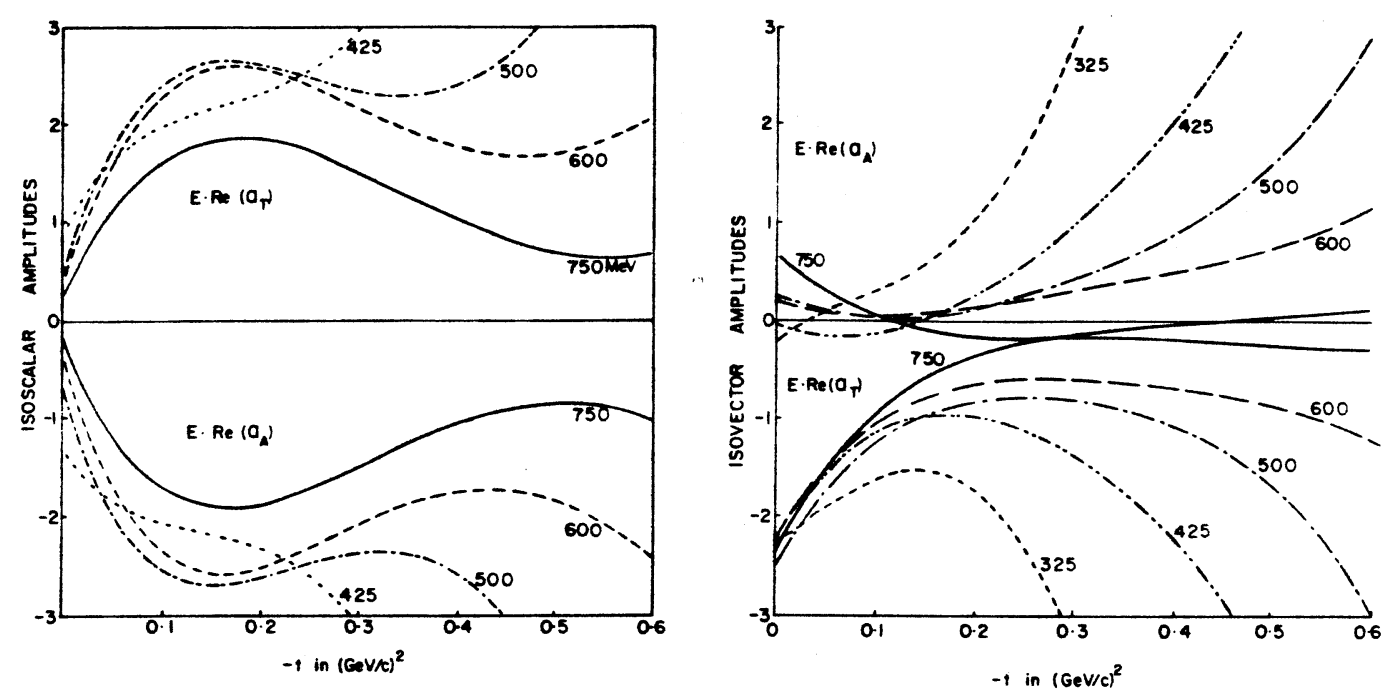

Fig. 3. The $a_{A}$ and $a_{T}$ amplitudes for different lab energies.

\section{The T-A and the V-S cancellations}

The name "strong interaction" should be used with reservation when applied to the $\mathrm{N}-\mathrm{N}$ interaction which is much weaker than the $\bar{N}-N$ interaction. The reason seems to be the presence of cancellations in the $\mathrm{N}-\mathrm{N}$ interaction. In Figs. 3 and 4 we depict the Fermi amplitudes $a_{T}$ and $a_{A}$. The cancellation of the isoscalar amplitudes is rather striking. This cancellation is also evident in the imaginary part of the isovector
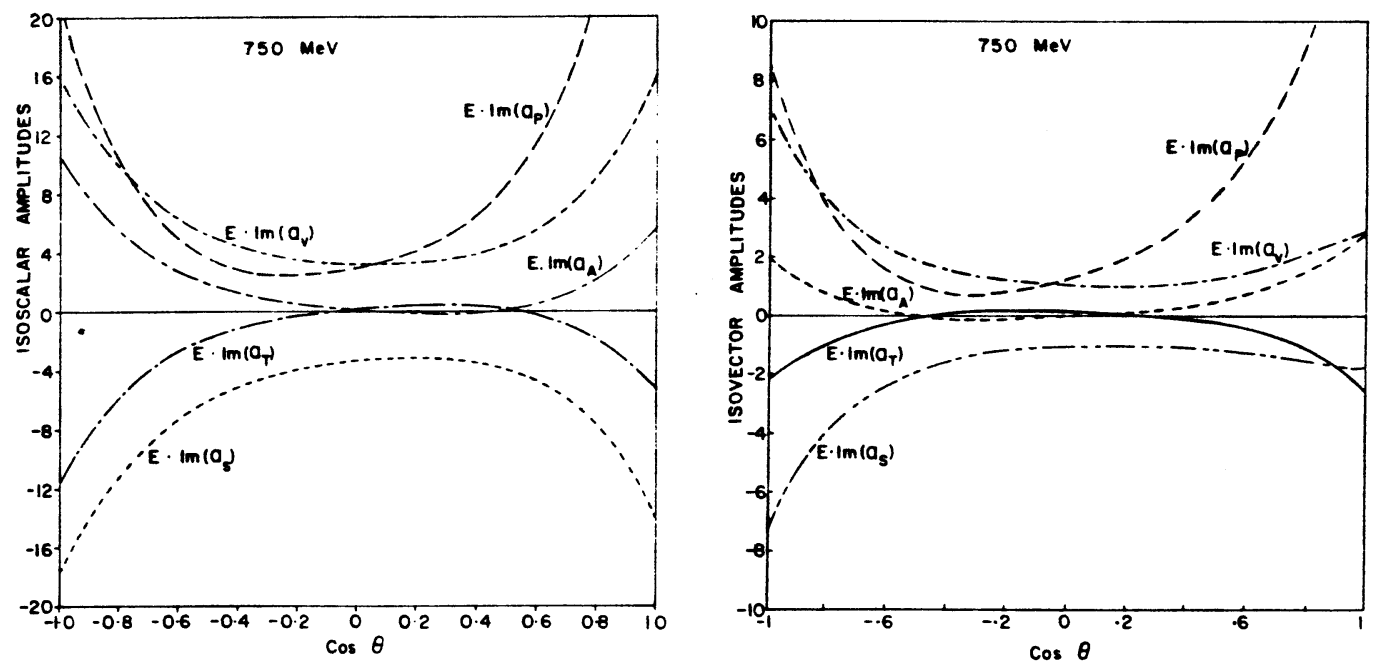

Fig. 4. The imaginary part of the $a_{S}, a_{V}, a_{T}, a_{A}$ amplitudes for $750 \mathrm{MeV}$. 
amplitudes, but seems to be absent in the forward direction of the real part. To our knowledge the origin of this T-A cancellation is not yet understood.

Let us compare the T-A cancellation with some of the consequences of isospin symmetry. As we approach the elastic threshold, isospin symmetry is strongly violated. As we increase the energy along the elastic cut the isopin symmetry "opens". The "opening" occurs much earlier in the imaginary part of the amplitudes than in the real part. A similar situation seems to occur for the T-A amplitudes, although the cancellation is not accurate enough to serve as a constraint on phase-shift analyses. In Fig. 5 the "opening" of the T-A cancellation is demonstrated for the imaginary part of the $a_{T}+a_{A}$ neutron-proton scattering amplitude.

In Figs. 6 and 4 the $a_{S}$ and $a_{V}$ amplitudes are depicted. The $a_{S}+a_{V}$ cancellation is less accurate than the $a_{T}+a_{A}$ cancellation and again is better satisfied for the imaginary parts and for the isoscalar amplitudes. The $V-S$ cancellation was advocated before by A.E.S. Green and coworkers in a potential model. 5

\section{Summary and discussion}

We have shown in Sec. IV that it is impossible to extract ten independent amplitudes having a definite exchange meson properties because five of them must be dependent. By taking combinations of the Fermi amplitudes it is possible to obtain some insight into the meson exchange content of the $\mathrm{N}-\mathrm{N}$ amplitudes. We defined two isospin combinations: the isoscalar and isovector amplitudes. By studying these amplitudes we can gain both qualitative and quantitative information about the $\mathrm{N}-\mathrm{N}$ interaction. For example, from Eq. (5.2) and Fig. 1 we can get estimates for the coupling constants

$$
\begin{aligned}
& g_{\varepsilon}^{2} / 4 \pi \simeq 16.4 m_{\varepsilon}^{2} /\left[m_{N}^{2} F_{\varepsilon}^{2}(0)\right], \\
& g_{\delta}^{2} / 4 \pi \simeq 1.2 m_{\delta}^{2} /\left[m_{N}^{2} F_{\delta}^{2}(0)\right], \\
&(1+f / g)_{\omega}\left(g_{\omega}^{2} / 4 \pi\right) F_{\omega}^{2}(0) / m_{\omega}^{2}+(1+f / g)_{\phi}\left(g_{\phi}^{2} / 4 \pi\right) F_{\phi}^{2}(0) / m_{\phi}^{2} \simeq 9.5 / m_{N}^{2} .
\end{aligned}
$$

From Fig. 2 we can get information about the $\pi \mathrm{NN}$ form factor. The results are uncertain to some extent because of the difficulty of taking out the p-meson contribution which simulates the two-pion-cut contributions. With the parameters of the vectormeson dominance model one obtains a form factor consistent with a nucleon bag radius of about $0.8 \mathrm{fm}$.

A new feature of the discussed amplitudes is the T-A cancellation in the isoscalar amplitudes and the T-A cancellation in the imaginary part of the isovector amplitudes. A less accurate cancellation is the scalar-vector one. The origin of these cancellations is not yet clear.

The existence of the $T-A$ and $S-V$ cancellations suggests probability that new relations or symmetries may apply to the $\mathrm{N}-\mathrm{N}$ interaction. In their present form the discussed cancellations are not accurate enough in order to be applied to reduce the number of independent amplitudes. One can look for further relationships. For example, related 


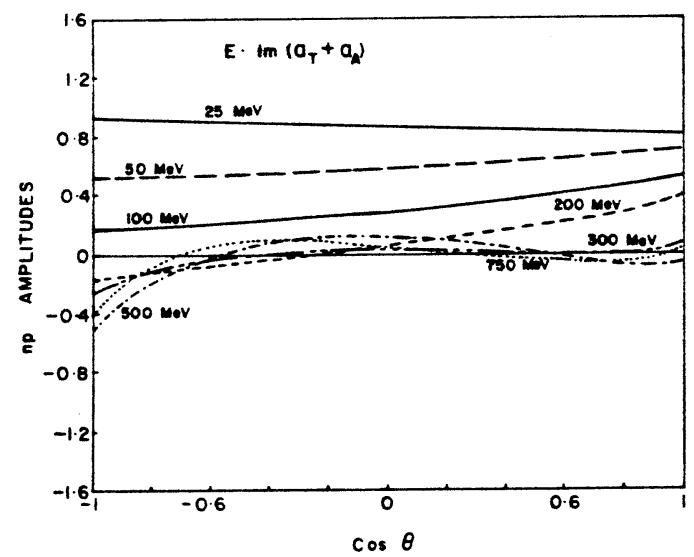

Fig. 5
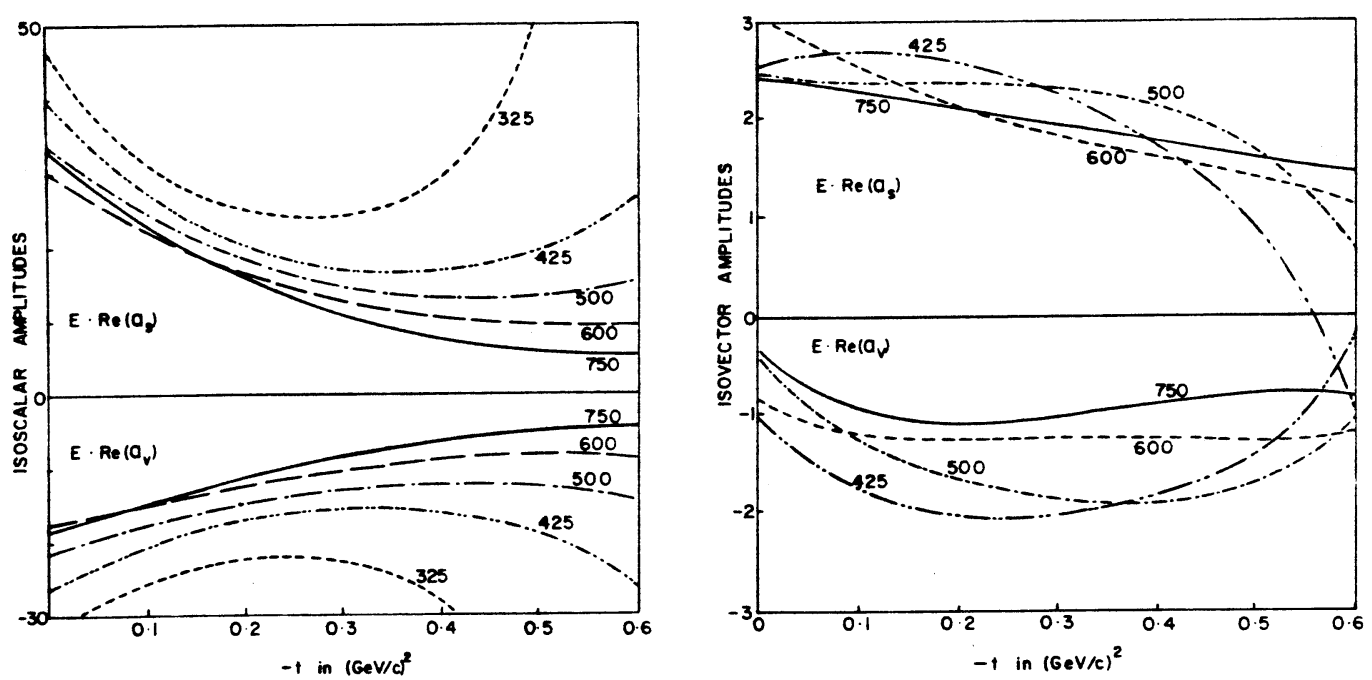

Fig. 6. The $a_{S}$ and $a_{V}$ amplitudes for different lab energies. 
to the T-A cancellation is the following amplitude

$$
a_{T}+\left(4 m^{2} a_{A}+t a_{p}\right) /\left(4 E^{2}-u\right) \text {. }
$$

If only direct Feynman diagrams and only meson exchanges of the so-called natural parity $\left(J^{P}=0^{+}, 1^{-}, 2^{+}, \ldots\right)$ are used, the amplitude (7.1) should vanish. Calculations show that this amplitude is rather small, but still not small enough to be a practical constraint. Another small amplitude (especially in the backward direction) is the real part of the following amplitude

$$
a_{T}(t)+a_{A}(t)+\frac{1}{2} a_{S}(u)+\frac{1}{2} a_{V}(u) \text {. }
$$

The examples of the amplitudes (7.1) and (7.2) demonstrate that the T-A cancellation can be an approximation to a more accurate relationship.

Apart from the existence of the cancellations the depicted amplitudes show the following unusual features:

(i) An unusual behaviour of the real part of the isoscalar ap amplitude. The change of sign to positive in Fig. 1 at higher energies, and the enormous magnitude, are somewhat puzzling. The maximum about $600 \mathrm{MeV}$ suggests correlation with $\Delta$-production or dibaryon resonances.

(ii) The isoscalar a amplitude is positive. This suggests that $f_{\omega} / g_{\omega}$ is positive, contrary to the usually accepted value -0.12 .

(iii) The isovector amplitudes $a_{V}$ and $a_{T}$ are relatively small, suggesting a rather small contribution of the $p$-meson.

(iv) The isovector $a_{T}$ amplitude is not consistent with natural parity meson exchanges. The constant value observed at $t=0$ suggests a meson exchange of non-natural parity and spin $J \geq 2$. A possible candidate is the $A_{3}$ meson.

(v) The resulting mass of the isoscalar exchange should be smaller than the w-meson mass.

It seems to us that the study of the Fermi amplitudes may lead to a better understanding of the properties of the $N-N$ interaction. More calculations, especially those including an error analysis, will be of utmost value.

I would like to acknowledge valuable discussions with A.W. Thomas and J.M. Richard.

\section{References}

1. R. Arndt and B.J. VerWest, in Proc. of Ninth Int. Conference on the Few-Body Problem, Eugene, Oregon, August 1980;

J. Bystricky, C. Lechanoine and F. Lehar, Saclay preprint DPhPE 79-01,0ctober 1979.

2. M.L. Goldberger et az., Phys. Rev. 120, 2250 (1960).

3. A. Gersten, Nucl. Phys. B12, 537 (1969).

4. G.A. Miller, A.W. Thomas and S. Théberge, Phys. Lett. $91 \mathrm{~B}, 192$ (1980) and TRIUMF preprint TRI-PP-80-8 (to be published in Phys. Rev. D)

5. A.E.S. Green and T. Sawada, Rev. Mod. Phys. 39, 594 (1967). 